\title{
EXPECTED UTILITY STRATEGIC DECISION MODELS FOR GENERAL INSURERS
}

\author{
By Danny SAmson \\ University of Melbourne
}

\begin{abstract}
It has been argued in previous studies that the expected utility decision criterion provides useful insights for certain insurance problems, such as underwriting, reinsurance and portfolio optimization problems. In this study three new models are developed which extend and generalize previous results. The first model analyses modified stop-loss reinsurance. The second model analyses risk pooling where both inward and outward reinsurance occur. Expected utility calculations can be used to provide insight on the attractiveness of competing reinsurance and risk pooling options. The third model is for strategic planning, where risk/reward tradeoffs for all the insurer's business activities (underwriting, investment, reinsurance) can be considered in aggregate. The simpler models can often be solved analytically however the strategic planning model is relatively complex and uses Monte Carlo techniques to determine retained earnings distributions. The expected utility approach has been found to be powerful, flexible and comprehensive as a decision aiding mechanism. From a normative viewpoint, this approach accounts very well for all the important decision elements. Recent developments in decision support systems will allow these models to be made available to practitioners in user friendly forms.
\end{abstract}

\section{INTRODUCTION}

A considerable volume of research has been conducted into the application of the expected utility decision criterion in risk and insurance. Much of the fundamental work was done by BORCH (1974) and important contributions were made by BuhlmanN (1971) and Freifelder (1979). SAmson and Thomas (1983) applied the criterion to reinsurance decision making and subsequently (SAMSON and Thomas, 1985) showed how such a decision criterion could be used as a screening device as well as a decision aiding tool.

In this study the earlier developments in using expected utility models for underwriting and reinsurance decision making are extended and generalized such as to facilitate comparisons of reinsurance and risk pooling. It is further argued that in general, from a normative standpoint these "risk position" decisions should not be taken in isolation of all other strategic decisions. Finally, a utility theory model is developed which encompasses all the strategic decisions of the insurer (underwriting, investment, reinsurance). 
MODEL 1: MODIFIED STOP-LOSS REINSURANCE

Deductibles and coinsurance agreements have become very popular in both personal and commercial lines of insurance. In reinsurance, modified stop-loss contracts (where a form of deductible exists) are also much used. In this section, upper bound premiums are developed for modified stop-loss reinsurance using the methods of SAMSON and THOMAS (1983). These principles, which allow a reinsurer to understand tradeoffs between retention levels and coinsurance proportions apply equally well to primary insurance where optimal deductible and/or coinsurance decisions can be made simultaneously.

Modified stop-loss reinsurance is a proportional transfer of risk (claims liability) above a specified retention. Hence it contains elements of both proportional and nonproportional reinsurance. In quota share reinsurance or stop-loss reinsurance there are only two primary parameters of interest, namely the premium and either the proportion ceded or retention level. All three of these elements enter into modified stop-loss reinsurance. Indeed quota share or pure stop-loss reinsurance forms can be defined as special cases of modified stop-loss reinsurance.

The advantages of modified stop-loss treaties for the insurer are in their flexibility and ability to allow the insurer to retain $100 \%$ of the claims liability for amounts below the retention level and cede portions above that level. For the reinsurer, an attractive feature of such treaties is the proportional participation of the insurer in large claim amounts (an advantage over pure stop-loss reinsurance). The need for the reinsurer to participate in small claims is obviated in modified stop-loss reinsurance (an advantage over quota share reinsurance).

\section{Expected Utility Upper Bound Premiums}

From the normative perspective of expected utility theory, the advantages of modified stop-loss reinsurance over quota share or stop-loss forms can be demonstrated. From SAMSON and Thomas (1983, p. 253) we can write the expected utility indifference condition for the insurer:

$$
E\left[U_{D}\left(A_{D}-P_{R M A X}-Y\right)\right]=E\left[U_{D}\left(A_{D}-X\right)\right]
$$

where claims $X$ are transformed by reinsurances into retained claims $Y . U_{D}$ is the utility function and $A_{D}$ represents all other assets. $P_{R M A X}$ is the upper bound reinsurance premium.

For quota share insurance:

$$
Y=F X \text { for all } X \text {, where } F \text { is the fraction retained. }
$$

For pure stop-loss reinsurance:

$$
\begin{array}{ll}
Y=X & \text { for } X \leqslant C \\
Y=C & \text { for all } X>C
\end{array}
$$

where $C$ is the retention limit. 
For modified stop-loss reinsurance:

$$
\begin{aligned}
& Y=X \text { for } X \leqslant C \\
& Y=C+F(X-C) \text { for } X>C
\end{aligned}
$$

where $C$ is the retention limit and $F$ is the fraction retained for $Y>C$.

To illustrate the upper bounds for modified stop-loss reinsurance we choose the exponential utility function $U(A)=-e^{-k A}$. This choice is based on its previous use by BorCH (1974), Freifelder (1979) and SAMSON and THOMAS (1983) and does not imply that its use is recommended without empirical justification. Indeed, as a matter of implementation a number of plausible utility functions should be considered and a new methodology for doing so is presented as an Appendix.

For this form of utility function, equation (1) becomes

$$
\begin{gathered}
\int_{0}^{c}-e^{-k\left(A_{D}-P_{R M A X}-Y\right)} \cdot f(Y) d Y+\int_{c}^{\infty}-e^{-k\left[A_{D}-P_{R M A X}-C+F(Y-C)\right]} \cdot f(Y) d Y \\
=\int_{0}^{\infty}-e^{-k\left(A_{D}-X\right)} f(X) \cdot d X
\end{gathered}
$$

This equation can be solved for any loss distribution although in some cases due to intractability, numerical methods may be necessary. Exact solutions can be found for the negative exponential loss function:

$$
f(x)=\lambda e^{-\lambda x}
$$

The solution to equation (3) for this case is

$$
P_{\text {RMAX }}=\frac{1}{k} \ln \left[\frac{1}{1+e^{(k-\lambda) C} \cdot[(k-k F) /(k F-\lambda)]}\right] .
$$

For the special case of quota share reinsurance, $C=0$ and equation (4) reduces to equation (22) in SAMSON and Thomas (1983, p. 259). For the special case of pure stop-loss reinsurance, $F=0$ and equation (4) reduces to equation (20) in SAMson and Thomas (1983, p. 258). Table 1 shows values of $P_{\text {RMAX }}$ for $k=$ $0.00005 \$^{-1}$ and $\lambda=\$ 1 / 2380.95$ (as used by SAMSON and Thomas (1983) and FREIFELDER (1979).

The insurer may have a large number of reinsurance possibilities available and the data in Table 1 (or else similar sets of data for whichever utility function form and parameters are appropriate) can be used to support those decisions. The calculated upper bound premiums are not market quotations, but represent the intrinsic value of the various reinsurance options to the insurer. The tradeoffs between proportional and nonproportional elements can be made by comparing alternatives with approximately equal upper bound values. For example, a maximum premium of $\$ 260$ applies to positions of:

1. Pure stop-loss reinsurance $(F=0)$ with a retention level of $C=\$ 6000$.

2. Approximately $F=0.56$ above $C=\$ 4000$.

3. Approximately $F=0.79$ above $C=\$ 2000$.

4. Approximately $F=0.90$ with $C=0$ (quota share reinsurance). 
TABLE 1

VALUES OF $P_{\text {RMAX }}$ FOR $k=0.00005, \lambda=1 / 2380.95$

\begin{tabular}{|c|c|c|c|c|c|c|}
\hline \multicolumn{7}{|c|}{ Retention Level $C$} \\
\hline $\begin{array}{c}F \\
\text { Fraction } \\
\text { Retained }\end{array}$ & 0 & 2000 & 4000 & 6000 & 8000 & 10,000 \\
\hline 0 & 2535 & 1169 & 549 & 260 & 123 & 58 \\
\hline 0.1 & 2295 & 1062 & 499 & 236 & 111 & 53 \\
\hline 0.2 & 2053 & 953 & 449 & 213 & 101 & 48 \\
\hline 0.3 & 1807 & 842 & 397 & 188 & 89 & 42 \\
\hline 0.4 & 1559 & 728 & 344 & 163 & 77 & 37 \\
\hline 0.5 & 1307 & 613 & 290 & 137 & 65 & 31 \\
\hline 0.6 & 1052 & 495 & 234 & 111 & 53 & 25 \\
\hline 0.7 & 794 & 375 & 178 & 84 & 40 & 19 \\
\hline 0.8 & 533 & 252 & 120 & 57 & 27 & 13 \\
\hline 0.9 & 268 & 127 & 60 & 28 & 13 & 6 \\
\hline 1.0 & 0 & 0 & 0 & 0 & 0 & 0 \\
\hline
\end{tabular}

These comparisons can similarly be made for any premium level.

A second mode of using this form of analysis is to compare market quoted premiums for various alternatives with calculated upper bound values both as a screening device (SAMSON and THOMAS, 1985) and as a method of ultimate choice.

A third mode is to obtain premium quotes and substitute them into the left side of equation (1) (or in the illustrative case, equation (3)) such as to evaluate the expected utility for each alternative.

Reinsurance agreements usually involve processes of negotiation between the insurer and a broker or reinsurer, hence the most valuable approach is likely to be the "upper bound" method. An insurer can quickly and efficiently know its preferences and determine its strategy if data such as in Table 1 is available during such negotiation processes. There would be potential advantages for the reinsurer to know the utility function of the insurer (as well as its own) so that it too could find an optimal negotiation strategy.

This analysis of modified stop-loss reinsurance can also be applied to primary insurance, where a potential insured is considering an optimal deductible $(C)$ and an optimal coinsurance level $(F)$. In most personal lines, $F=0$ (there is no coinsurance element above the deductible) however risk managers often need to make decisions involving tradeoffs between $C, F$ and $P$ (premiums) in commercial lines. The present analysis generally applies to aggregate loss distributions (i.e., implicitly assumes only one claim or an aggregation of losses from many claims) and can be modified to incorporate claim frequency distributions.

\section{MODEL 2: RISK POOLING}

BorCH $(1974$, p. 25) has commented that:

An insurance company generally wants to retain as much of its portfolio as possible. If it is not obliged to reinsure to satisfy government 
requirements, a company will not usually give a part of its portfolio away to a reinsurer unless it gets another more or less equivalent portfolio in return.

The advantages of risk pooling, sharing or reciprocal agreements are principally that in such cases the net premium base of the insurer is eroded less than in the case of pure outward reinsurance.

For reciprocal reinsurance or risk pools, the expected utility of entering the risk pool is:

$$
E\left[U_{D}\left(A_{D}-Y-Z-\Delta P\right)\right]
$$

where $Z$ is the inward claims liability and $\Delta P$ is the net premium adjustment (outward-inward).

In general the insurer wishes to maximize expression (5). Pure outward cession occurs where $Z=0$ and $\Delta P=P_{R}$ (the outward reinsurance premium). If $Y$ and $Z$ are statistically independent then expression (5) can be evaluated for various alternatives of $Y$ and $Z$ as:

$$
\int_{0}^{\infty} \int_{0}^{\infty} U_{D}\left(A_{D}-Y-Z-\Delta P\right) f(Y) \cdot d Y f(z) \cdot d Z
$$

In most cases this integral will not be analytically tractable, and numerical methods involving risk analysis are recommended. If $Y$ and $Z$ are statistically dependent then the attractiveness of risk pooling is altered. If the covariance of $Y$ and $Z$ is high relative to the variances of $Y$ and $Z$ then risk pooling may be unattractive, and certainly may be worse than pure outward reinsurance. This can be demonstrated using normally distributed claims distributions (see HoGG and Klugman, 1984, p. 35 for a discussion of this distribution in the insurance claims context), and assuming that mean-variance efficiency is desired.

For an $\alpha$ risk pool, ${ }^{*}$ a point of minimum variance exists for the pool only if the covariance of the claims distributions is less than the average of their variances.

Prior to pooling, let the claims variance of participating companies 1 and 2 be $\sigma_{1}^{2}$ and $\sigma_{2}^{2}$ respectively.

For company 1, risk pooling transforms its claim variance to

$$
\sigma_{1}^{2^{*}}=(1-\alpha)^{2} \sigma_{1}^{2}+\alpha^{2} \sigma_{2}^{2}+2(1-\alpha)(\alpha) \sigma_{12} .
$$

At critical points, $\delta \sigma_{1}^{2^{*}} / \delta \alpha=0$.

$$
\frac{\delta \sigma_{1}^{2^{*}}}{\delta \alpha}=-2(1-\alpha) \sigma_{1}^{2}+2 \alpha \sigma_{2}^{2}+(2-4 \alpha) \sigma_{12} .
$$

The second derivative is given by:

$$
\frac{\delta^{2} \sigma_{1}^{2^{*}}}{\delta \alpha^{2}}=2 \sigma_{1}^{2}+2 \sigma_{2}^{2}-4 \sigma_{12}
$$

\footnotetext{
*An insurer forms an " $\alpha$ risk pool" when it lays off a proportion $\alpha$ of its risk liability and accepts the same proportion $\alpha$ from another party (which may be another insurer, reinsurer or a multi-party pool). The term $\sigma_{1}^{2^{*}}$ denotes variance including the effects of risk pooling.
} 
Hence:

$$
\sigma_{12}<\frac{\sigma_{1}^{2}+\sigma_{2}^{2}}{2} \text { for a minimum. }
$$

The minimum variance (found by equating expression (7) to zero) occurs at

$$
\alpha^{*}=\frac{\sigma_{1}^{2}-\sigma_{12}}{\sigma_{1}^{2}+\sigma_{2}^{2}-2 \sigma_{12}} .
$$

If this minimum is to exist in the range $0 \leqslant \alpha^{*} \leqslant 1$ then:

$$
\frac{\delta \alpha_{1}^{2^{*}}}{\delta \alpha} \leqslant 0 \quad \text { at } \alpha=0 \text { and } \quad \frac{\delta \alpha_{1}^{2}}{\delta \alpha} \geqslant 0 \quad \text { at } \alpha=1
$$

implying additional conditions $\sigma_{1}^{2} \geqslant \sigma_{12}$ and $\sigma_{2}^{2} \geqslant \sigma_{12}$.

Note that if $\sigma_{1}^{2}=\sigma_{2}^{2}$ then the critical point is at $\alpha^{*}=\frac{1}{2}$ regardless of $\sigma_{12}$. This critical point may however be a maximum, minimum or stationary point depending on the value of expression (8).

To facilitate a comparison of risk pooling and modified stop-loss reinsurance, we calculate upper bound premiums in the case of exponential utility functions, i.e., for the equation:

$$
\int_{0}^{\infty} \int_{0}^{\infty}-e^{-k_{D}\left(A_{D}-Y-Z-P\right)} f(y) \cdot d Y \cdot f(z) \cdot d z=\int-e^{-k_{D}\left(A_{D}-X\right)} f(x) d x
$$

where $X$ is the original claims distribution of the insurer which is negative exponential with parameter $\lambda_{1}, Y$ is the retained claims of $X ; Y=\alpha X ; Z$ is the original claims distribution of the other party, and the insurer accepts a proportion $(1-\beta)$ of this risk. $Z$ is assumed negative exponential with parameter $\lambda_{2}$. The solution to equation (11) is

$$
P=\frac{-1}{k} \ln \left[\frac{\left(k(1-\beta)-\lambda_{2}\right)\left(k \alpha-\lambda_{1}\right)}{\lambda_{2}\left(k-\lambda_{1}\right)}\right]
$$

and if $\lambda_{2}=\lambda_{1}=1 / \$ 2380.95$ and $k=0.00005$ as before, the upper bound net premiums are shown in Table 2 . Note that the solution (12) generally applies to the range $k<\lambda_{1}$. Implicit in this illustration is the assumption of independence between $X$ and $Z$ and hence between $Y$ and $Z$.

A number of observations can be made about the data in Table 2 and about comparisons between Table 2 and Table 1. First, there is symmetry in Table 2 across the non-leading diagonal. The insurer is indifferent (only in cases where the inward and outward claims density functions are identical) between positions of (outward, inward) proportions of $(\alpha, \beta)$ and $(1-\beta, 1-\alpha)$ for any $\alpha$ and $\beta$ pair. Secondly $P$ is not zero in Table 2 for cases where $\alpha=\beta$ unless $\alpha=\beta=0$ or $\alpha=\beta=1$ (in which cases no effective change occurs). For cases where $\alpha$ and $\beta$ are equal but not 0 or $1, P$ is positive, indicating that for a risk averse 
TABLE 2

VAlues of $P_{\text {RMax }}$ fOr a Two PARTy RISK POOL

\begin{tabular}{|c|c|c|c|c|c|c|c|c|c|c|c|}
\hline \multicolumn{12}{|c|}{$\alpha$} \\
\hline $\boldsymbol{\beta}$ & 0 & 0.1 & 0.2 & 0.3 & 0.4 & 0.5 & 0.6 & 0.7 & 0.8 & 0.9 & 1.0 \\
\hline 0 & 0 & -240 & -482 & -728 & -976 & -1228 & -1483 & -1741 & -2002 & -2267 & -2536 \\
\hline 0.1 & 268 & 28 & -214 & -459 & -708 & -959 & -1214 & -1472 & -1734 & -1999 & -2267 \\
\hline 0.2 & 533 & 293 & 51 & -194 & -443 & -695 & -949 & -1207 & -1469 & -1734 & -2002 \\
\hline 0.3 & 794 & 555 & 312 & 67 & -181 & -433 & -688 & -946 & -1207 & -1472 & -1741 \\
\hline 0.4 & 1052 & 813 & 570 & 325 & 77 & -175 & -430 & -688 & -949 & -1214 & -1483 \\
\hline 0.5 & 1307 & 1068 & 825 & 580 & 331 & 80 & -175 & -433 & -695 & -959 & -1228 \\
\hline 0.6 & 1559 & 1319 & 1077 & 831 & 583 & 331 & 77 & -181 & -443 & -708 & -976 \\
\hline 0.7 & 1807 & 1568 & 1325 & 1080 & 831 & 580 & 325 & 67 & -194 & -459 & -728 \\
\hline 0.8 & 2053 & 1813 & 1571 & 1325 & 1077 & 825 & 570 & 312 & 51 & -214 & -482 \\
\hline 0.9 & 2295 & $\cdot 2055$ & 1813 & 1568 & 1319 & 1068 & 813 & 555 & 293 & 28 & -240 \\
\hline 1.0 & 2535 & 2295 & 2053 & 1807 & 1559 & 1307 & 1052 & 794 & 533 & 268 & 0 \\
\hline
\end{tabular}

$\alpha=$ proportion retained of outward risk transfer.

$1-\beta=$ proportion accepted of inward risk transfer.

$\lambda_{2}=\lambda_{1}=1 / 2380.95$.

$k=0.00005$.

insurer there is a considerable advantage from forming equitable risk pools. For cases where $\alpha=\beta, P$ takes on a maximum value at $\alpha=\beta=0.5$.

\section{Implementation: Aided Decision Making Using Expected Utility Models}

An insurer may have a number of alternatives open to it as methods of modifying its claims liability position, including various reinsurance and risk pool opportunities. Each of these may involve a number of parameters (retention limits, proportions retained, premiums, etc.) and comparisons (to determine the best option) cannot be accurately made without reducing the effects of these complex choices to a single measure. In decision theory, one useful procedure for comparing alternatives is to identify the one with the highest expected utility, however practical difficulties may preclude such processes from directly occurring in negotiation sessions because of the extensive calculations which are required. For example, with only a two party risk pool and tractable distributions and utility functions, the left hand side of equation (11) has to be calculated. More generally, numerical solutions are often required. The upper bound approach can be used to produce tables which can be readily used in evaluating reinsurance and risk pooling options.

As an illustration, consider an insurer (with exponential utility, $k=0.00005$ ) which is considering the following five options on its claims liability (which has exponential claims probability density function and $\lambda=1 / \$ 2380.95$ ).

1. Modified stop-loss reinsurance of $50 \%$ retention above a limit of $\$ 2000$, costing a premium of $\$ 700$.

2. Modified stop-loss reinsurance with $80 \%$ retention above a retention of $\$ 4000$ costing $\$ 118$. 
3. Forming a risk pool with another party having an identical claims liability where the fraction retained $\alpha=0.8$ and the fraction accepted $(1-\beta)=0.2$. There is no premium.

4. Entering a risk pool with another party having an identical claims liability where $\alpha=0.8,1-\beta=0.1$ and the premium charged is $\$ 300$.

5. Entering a risk pool where the other party has an exponential claims density function with parameter $\lambda_{2}=\lambda_{1} / 2$. For this pool the proposed retained claims on the outward risk is $\alpha=0.8$ and the fraction accepted on the inward risk is $1-\beta=0.3$ with net (inward) premium of $\$ 860$.

For option 1, the upper bound premium from Table 1 is $\$ 613$, hence option 1 can be immediately screened out as being unattractive in an absolute sense (this reinsurance option is worse than no reinsurance).

For option 2, the upper bound premium is $\$ 120$, hence this option is slightly better than the original "no reinsurance" position.

Option 3 has an upper bound premium of $\$ 51$ and since this is a pooling of identical risks, no premium need be paid and a significant advantage can be gained. Note that in this situation of identical risks, a $20 \%$ sharing of risks provides a benefit of over $60 \%$ of the maximum (a $\$ 51$ equivalent gain when $\alpha=\beta=0.2$ compared to a maximum of $\$ 80$ when $\alpha=\beta=0.5$ ).

Option 4 has an outward premium which is above the upper bound ( $\$ 293$ from Table 2) and is rejected.

For option 5 the indifference premium is a lower bound on an inward premium, and is $-\$ 949$ (calculated in the same manner as for options 3 and 4) meaning that an inward payment of at least $\$ 949$ must occur to make this option worthwhile. Since the premium offered is only $\$ 860$, this offer is rejected.

In conclusion the most attractive option is 3 and it would be to the advantage of the insurer to increase the mutual participation in the risk pool from the suggested $20 \%$ up to $50 \%$. Had more than one option passed the screen and been attraetive in an absolute sense, the expected utility of these options could be calculated and compared.

A number of other important implementation issues exist, including estimation problems and problems of intractability. The expected utility model inputs are the claims density functions and the utility functions. Much work and many advances have occurred in estimation for both these elements, for example, HoGG and KLUGMaN (1984) on loss distribution estimation, and FarQuHAR (1984) and SAMSON (1984) on utility function assessment. Although inaccuracy always exists in these empirical procedures, improved methods are continuously increasing the ability of researchers and practitioners in fitting functions to claims and utility functions.

In more complex reinsurance and risk policy arrangements than those illustrated above, analytical solutions to the equations cannot generally be found and either numerical integration techniques or Monte Carlo procedures can be used to give close approximation solutions. Monte Carlo procedures are particularly powerful and flexible, and can account for correlations between variables (HERTz 
and Thомas, 1983). These techniques also readily allow for sensitivity analysis to be performed. Monte Carlo methods would be most suitable in complex multi-party risk pools or layered reinsurance arrangements, where direct integration of the appropriate equations would be difficult or impossible. In such cases the Monte Carlo techniques would be used to approximate total claims distributions and the expected utility function can then be numerically applied to the total claims function.

MODEL 3: TOWARDS A GENERAL THEORY OF INSURANCE

Utility theory provides a useful basis for modelling the activities of an insurer such as to produce the best overall or aggregate result. Consider an insurer with assets $A_{t}$ at time $t$, which must make decisions regarding underwriting, investment, reinsurance and other functions such that at time $t+1$ its assets will be $A_{t+1}$, where $A_{t+1}=A_{t}+\Delta A$. Although accounting practices vary considerably across insurers particularly in an international context we develop a simplified but representative decomposition of $\Delta A$ below based on a number of balance sheets.

$$
\Delta A=\Delta C+\Delta R+R E
$$

where $\Delta C$ is a change in paid up capital, $\Delta R$ is a change in reserves (share premium, asset revaluation, surplus, etc.), $R E$ is retained earnings.

One simplified decomposition for $R E$ is:

$$
R E=(1-T)\left[\sum\left(P_{i}-X_{i}-E_{i}\right)+R+P I+R e\right]-D
$$

$\boldsymbol{P}_{i}=$ premiums in line $i$,

$X_{i}=$ claims in line $i$,

$E_{i}=$ expenses in line $i$,

$R=$ investment returns,

$P I=$ profit $/$ loss on sale of investments,

$R e=$ reinsurance effects (premiums and claims, both outward and inward), and

$D=$ dividends paid,

$T=$ representative tax rate.

Most companies' retained earnings equations contain many other terms, however the primary operating effects are contained in equation (13).

In maximizing the expected utility of the insurer's aggregate earnings, risk/reward decisions within particular insurance accounts or investment decisions are evaluated in terms of their effect on the firm as a whole. Figure 1 shows the flow diagram for the computerized decision support system on which this model has been implemented. For any set of strategic variables, Monte Carlo methods were used based on probabilistic inputs about premium volumes, claims, expenses and investments to calculate a probability distribution of retained 


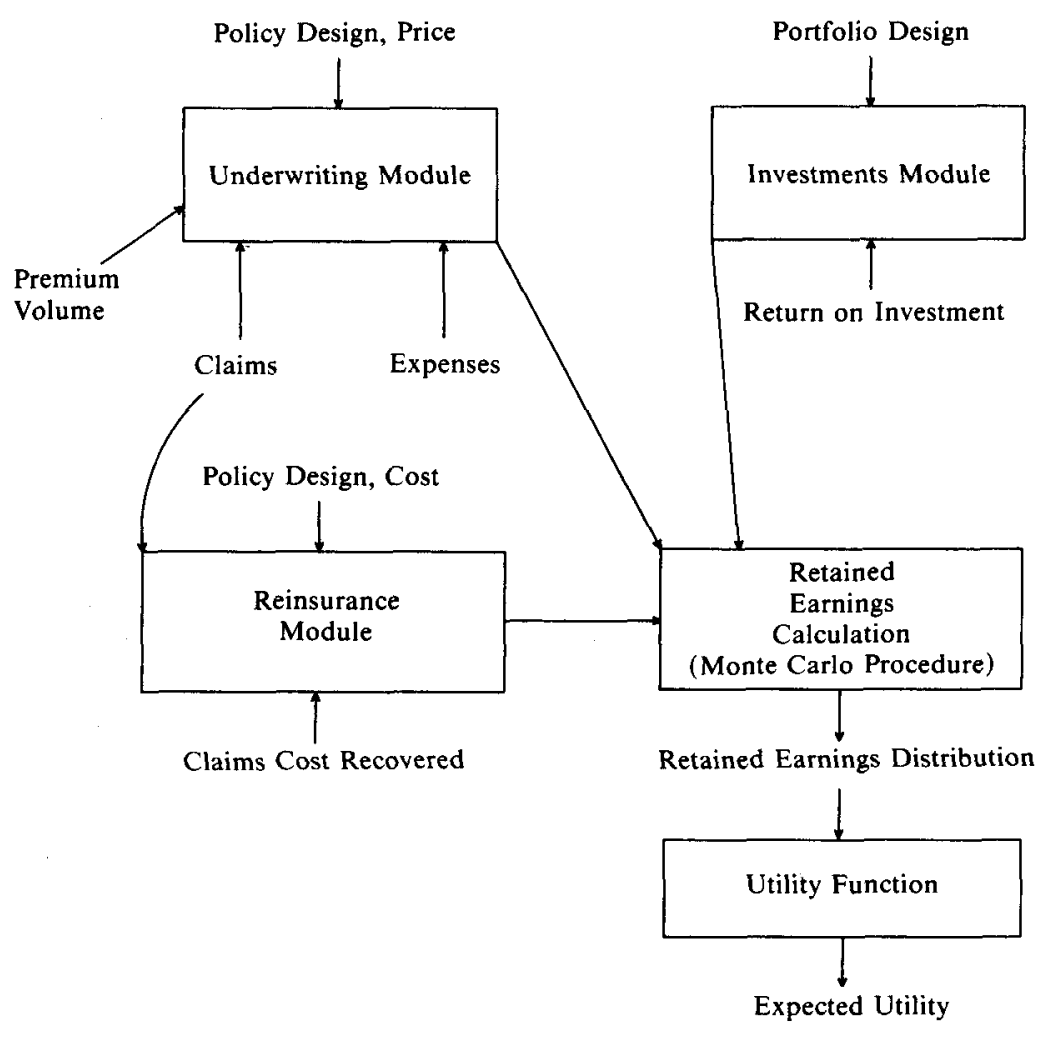

FIGURE 1

earnings. A utility function, with form and parameters identified using the method in the Appendix, is applied to this output distribution, and expected utilities can be calculated and compared for a large number of potential strategies in a short time.

The model can be used for generalized strategic planning, single unit changes (such as entering a new market or line of business), or fine tuning. This normative model of insurer management does not in itself constitute a general theory of insurance, but provides decision makers with important insights regarding optimal operating strategies.

The reinsurance models in SAMSON and THOMAs (1983) considered reinsurances in isolation of other important decision variables such as underwriting and investment. Although in some circumstances this may be reasonable, the present approach generalizes the use (and hence increases the power) of expected utility models such that underwriting, investment and reinsurance problems can be solved simultaneously.

KEENEY (1982) suggested that the aim of decision analytic models is to provide insights not answers, and similarly the aim of decision support systems and 
models is to aid decision and judgement processes and not replace them. The system can be used to provide insight and evaluate decisions such as:

1. Increasing (decreasing) claim adjustment efforts as a means of reducing (increasing) claims. Secondary effects such as changes in cash flow for investments and reinsurance premiums can be automatically accounted for by the system.

2. Underwriting new business, including effects on profit using various reinsurance agreements [based on the developments in SAMSON and THOMAS (1983)] and including reserving and investment policy effects.

3. Changing premium structures for existing business, in which case estimates of demand elasticities are required. Insights on changing premium structures can best be gained by considering the many secondary effects (as well as primary demand effects). In this decision context, the effects of changing premiums cause nearly every parameter shown on Figure 1 to change and the aggregate effect of such changes can be calculated using an expected utility approach.

This generalized strategic planning model can be implemented as a decision support system. It was developed at the University of Illinois as a user-friendly interactive system capable of being implemented on micro or mainframe computers. The user inputs a variety of data on claims distributions, investment returns, reinsurance options, etc., and can iterate with the system and evaluate various strategies in this computer aided manner, eventually converging upon a satisfactory (and hopefully optimal) solution. The user friendly character of such systems allow top management to participate in the creative, structuring processes and sensitivity analyses rather than merely to choose between previously structured options.

Decision support systems can also be artificially intelligent. In this system a useful feature is its ability to suggest a strategic response (or at least a direction of response) to a change in an exogenous variable. For example, if general market prices begin to move in a particular direction, the system could consider the effect of various options not only on underwriting profit but also on investments, reinsurances and ultimately on the retained earnings distribution.

\section{DISCUSSION OF THE USE OF EXPECTED UTILITY MODELS}

Many researchers have suggested that expected utility could be used in insurance. BuHLMANN (1971) suggested a "principle of zero utility" premium calculation method and FreIFELDER (1979) proposed exponential utility ratemaking rules. BORCH (1974) usefully applied utility theory to a number of aspects of insurance. None of these studies, however, proposed a unifying insurance theory which involved both a decision aiding mechanism (the structuring and evaluation model of Figure 1) and an axiom based (see KEENEY, 1982) objective function (i.e., maximize expected utility) which would use this approach to simultaneously set effective strategies for all of the insurer's activities.

The strength of the proposed approach is its ability to relate decisions such as reinsurance and investment decisions to an aggregate "whole firm" context. Many 
other approaches have been suggested for insurer management including risk theory and portfolio theory. From the viewpoint of expected utility analysis, we find these approaches to be merely special cases involving assumptions on the form of the utility function. Risk of ruin theory can be interpreted as involving a two state utility function (Roy, 1953, p. 432) which is impractical and generally unrealistic. Portfolio theories usually assume either quadratic utility or joint normally distributed returns which, particularly in an insurance context, do not seem to be justifiable. Cummins and NyE (1981) used a utility theory approach to indicate optimal operating positions on a mean-variance efficient frontier. While this approach is a useful one, the assumption that utility can be expressed in terms of mean and variance may not be appropriate in all cases. The present (Monte Carlo) approach has the advantage of being completely flexible with regard to distributional forms. We believe that many returns distributions in insurance companies are skewed (HoGG and KLUGMAN, 1984) and/or truncated by reinsurances (SAMSON and THOMAS, 1983).

\section{CONCLUSIONS}

First, since it has been demonstrated that quota share and stop-loss reinsurances are merely special cases of modified stop-loss reinsurance, insurers should be aware that optimal solutions need not generally occur when fractions retained $(F)$ or retention limits $(C)$ are zero. Modified stop-loss reinsurance forms with $F \neq 0$ and $C \neq 0$ have advantages over the special cases for both insurers and reinsurers and the expected utility solutions can be used to aid decision makers.

Second, risk pooling has the advantage (over pure outward reinsurance) of achieving risk diversification while not eroding the premium base of the insurer. An expected utility model was developed for risk pools which is generally able to evaluate competing alternatives. Through such analyses, pure reinsurance can be viewed as a special case of risk pooling [where no inward risk transfer occurs, i.e., $Z=0$-in equation (6)]. A risk pool involving two idential risks is shown to reduce variance only if the covariance of the claims distribution is less than the average of the variances.

Third, in generalized strategic planning models, alternative options are evaluated in terms of their aggregate effect; only rarely is it reasonable to make decisions in isolation. The expected utility model can be generalized to encompass underwriting, risk transfer and investment actions, with the expected utility of strategy sets being found using numerical (Monte Carlo) methods. This model's utility is its capability of relating decisions involving individual business units of the insurer to the context of the "firm as a whole".

\section{REFERENCES}

BORCH, K. (1974) The Mathematical Theory of Insurance. Lexington Books: Lexington, Mass. Buhlmann, H. (1971) Mathematical Methods in Risk Theory. Springer-Verlag: London. Carter, R. (1979) Reinsurance. Kluwer: London. 
Cummins, J. D. and Nye, D. J. (1981) Portfolio Optimization Models for Property Liability Insurance Companies: An Analysis and Some Extensions. Management Science 27 (4), 414-430.

Farquhar, P. H. (1984) Utility Assessment Methods. Management Science 30 (11), 1283-1300.

Freifelder, L. (1979) Exponential Utility Theory Ratemaking: An Alternative Ratemaking Approach. Journal of Risk and Insurance 46 (3).

HerTZ, D. B. and Thomas, H. (1983) Risk Analysis and Its Applications. Wiley: New York.

HoGg, R. V. and KLUgmaN, S. A. (1984) Loss Distributions. John Wiley: New York.

KeENEY, R. L. (1982) Decision Analysis: An Overview. Operations Research 30 (5), 803-838.

RoY, A. D. (1953) Safety First and the Holding of Assets. Econometrica 431-449.

SAMSON, D. A. and ThOMAS, H. (1983) Reinsurance Decision Making and Expected Utility. Journal of Risk and Insurance 50 (2), 249-264.

Samson, D. A. and Thomas, H. (1985) Decision Analysis Models in Reinsurance. European Journal of Operational Research 19 (2), 201-211.

Samson, D. A. (1984) Utility Function Assessment using Unrelated Gambles. Working Paper, University of Illinois.

\section{APPENDIX: A UTILITY FUNCTION FITTING METHODOLOGY}

The computer program elicits from the decision maker a set of certainty equivalents to gambles for use in fitting a utility function. These gambles are of the form:

$$
\left(X_{i}, P_{i}, Y_{i}\right) \sim C_{i}
$$

where $C_{i}$ is the certainty equivalent of a gamble involving a probability $P_{i}$ of outcome $X_{i}$ and a probability $\left(1-P_{i}\right)$ of an outcome $Y_{i}$. The decision maker can specify any three of these four parameters (or have the system to do so) and make a subjective judgment about the fourth. Single parameter utility functions (such as the commonly used exponential and logarithmic) can be fitted to the data from just one gamble, however the function would be highly sensitive to judgment errors under such conditions. To minimize the affect of these random errors, $N$ gambles are used and random errors are assumed to be independent. Hence if judgments on $C_{i}$ are made, errors $\left(e_{i}\right)$ are given by:

$$
U\left(C_{i}+e_{i}\right)=P_{i} U\left(X_{i}\right)+\left(1-P_{i}\right) U\left(Y_{i}\right)
$$

or

$$
e_{i}=-C_{i}+U^{-1}\left[P_{i} U\left(X_{i}\right)+\left(1-P_{i}\right) U\left(Y_{i}\right)\right] \text {. }
$$

The system can find least square error fits of various utility functions and recommends the use of that function which has the least square total error.

For the exponential utility function

$$
U(X)=\frac{1}{r}\left(1-e^{-r X}\right)
$$

we find

$$
e_{i}=-C_{i}-\frac{1}{r} \ln \left[P_{i} e^{-r X_{i}}+\left(1-P_{i}\right) e^{-r Y_{i}}\right]
$$

For the logarithmic utility function,

$$
U(X)=\ln (X+k)
$$


$e_{i}$ is given by:

$$
e_{i}=-C_{i}-k+e^{\left[P_{i} \ln \left(X_{i}+k\right)+\left(1-P_{i}\right) \ln \left(X_{i}+k\right)\right]} .
$$

These methods have distinct advantages over traditional utility fitting methodologies in that:

1. "Chaining" of gambles is not used in this method, hence biases are not propagated and compounded as in other methods.

2. The "range effect" is eliminated because the decision maker can specify any values of $X_{i}$ and $Y_{i}$ that are convenient.

3. By carefully choosing values of $X_{i}, Y_{i}$ and $P_{i}$ to be relevant to the types of decisions being made, other biases such as the certainty effect and the probability effect can be minimized.

DANNy SAMPSON

Graduate School of Management, University of Melbourne, 3052 Parkville, Victoria, Australia 